Pathologe $2007 \cdot 28: 291-293$

DOI 10.1007/s00292-006-0874-6

Online publiziert: 15. November 2006

(c) Springer Medizin Verlag 2006

\author{
U. Güth ${ }^{1} \cdot$ G. Singer ${ }^{2}$ \\ ${ }^{1}$ Frauenklinik, Universitäts-Spital Basel, Schweiz \\ 2 Institut für Pathologie, Universitäts-Spital Basel, Schweiz
}

\title{
Ektopes Prostatagewebe in der Cervix uteri
}

\section{Fallbericht \\ Anamnese, klinischer Befund und Therapie}

Die Hospitalisation der 82-jährigen $\mathrm{Pa}$ tientin erfolgte wegen einer Thrombose der linken V. femoralis communis. Im Rahmen der klinischen Diagnostik wurde in einem gynäkologischen Konsilium ein Unterbauchtumor diagnostiziert. Bei den daraufhin durchgeführten bildgebenden Untersuchungen fand sich sonographisch und in der CT im kleinen Becken eine $10 \times 15 \mathrm{~cm}$ große glatt begrenzte, mehrkammerige, inhomogen echoarme Raumforderung, welche die V. cava inferior komprimierte. Der Tumormarker CA 125 war mit $81 \mathrm{U} / \mathrm{ml}$ erhöht (Norm: $<35 \mathrm{U} / \mathrm{ml}$ ).

Weitere Punkte in der gynäkologischen Anamnese:
- Zustand nach 2 Spontangeburten,

- keine gynäkologischen Voroperationen,

- keine Hormontherapie.

Bei Verdacht auf ein Ovarialkarzinom wurde eine Längslaparotomie durchgeführt. Bei dem Eingriff erfolgten eine Hysterektomie, die beidseitige Adnexektomie und die Omentektomie. Im intraoperativen Schnellschnitt wurde histologisch
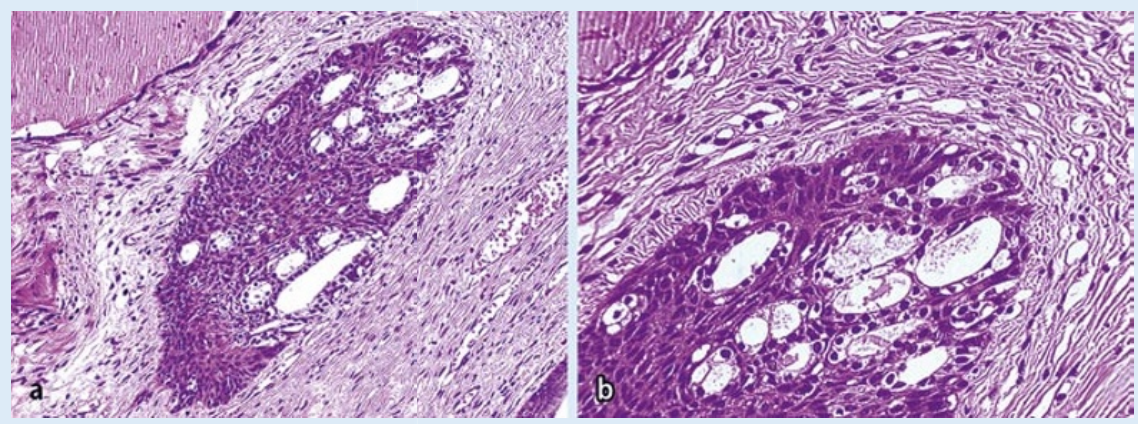

Abb. 1 A Ektopes Prostatagewebe in der Zervix uteri. a Übersichtsaufnahme. Typische kribriforme Architektur aus Drüsen mit umgebender plattenepithelartiger Proliferation von Basalzellen (HE-Färbung, Vergr. 200:1). b Detailaufnahme. Die glandulären Zellen zeigen ein vakuoläres Zytoplasma (HE-Färbung, Vergr. 400:1)
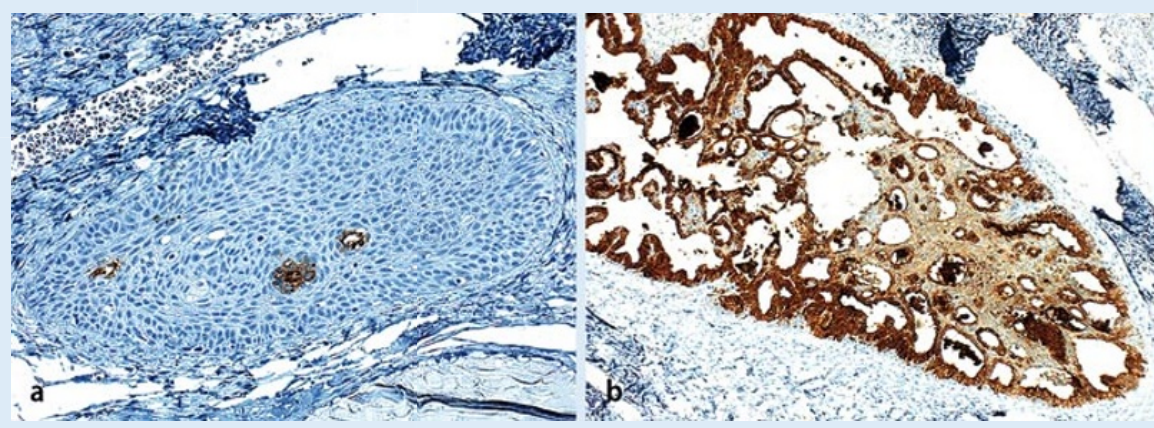

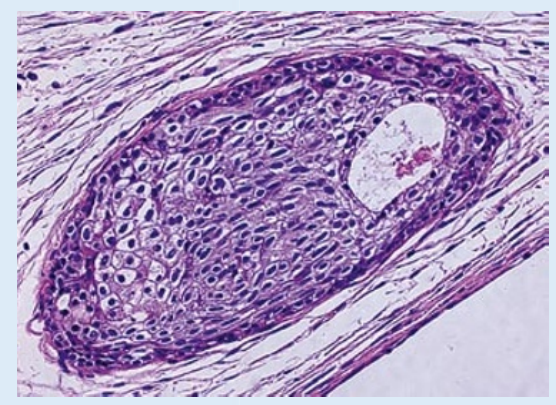

Abb. $2 \Delta$ Ein zweiter Herd mit überwiegend plattenepithelartiger Komponente (HE-Färbung, Vergr. 200:1)

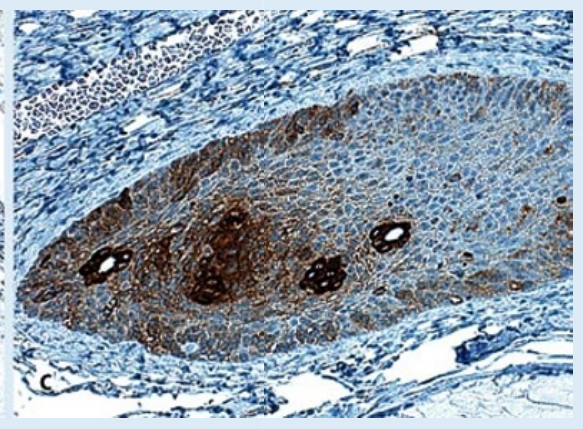

Abb. 3 A Immunhistochemische Untersuchungen. a, b Prostataspezifisches Antigen (PSA; Vergr. 200:1). In der Schnittstufe zu Teilabb. a zeigte sich ein größeres glanduläres Areal. c Prostataspezifische alkalische Phosphatase (PSAP;Vergr. 200:1) 
Pathologe 2007· 28:291-293

DOI 10.1007/s00292-006-0874-6

(C) Springer Medizin Verlag 2006

\section{U. Güth · G. Singer \\ Ektopes Prostatagewebe in der Cervix uteri}

\section{Zusammenfassung}

Ektopes Prostatagewebe ist ein selten beobachtetes Phänomen im unteren Genitaltrakt der Frau. Ätiologisch werden Entwicklungsanomalien, eine Metaplasie vorbestehender endozervikaler Drüsen und Reste embryonaler Genitalanlagen diskutiert. Wir berichten über ektopes Prostatagewebe in der Cervix uteri einer 82-jährigen Patientin. Dieses Gewebe fand sich als Zufallsbefund in einem Hysterektomiepräparat. Diese ungewöhnliche Läsion sollte besonders von atypischen glandulären Läsionen der Cervix uteri abgegrenzt werden. Immunhistochemische Zusatzuntersuchungen sichern die Diagnose.

\section{Schlüsselwörter}

Cervix uteri · Prostatagewebe - Ektopie · Entwicklungsanomalie $\cdot$ Immunhistochemie

\section{Ectopic prostatic tissue in the uterine cervix}

\begin{abstract}
Ectopic prostate tissue is a rare occurrence in the lower female genital tract. Possible theories of histogenesis include a developmental anomaly, metaplasia of preexisting endocervical glands, and derivation from mesonephric remnants. We report an example of prostatic tissue involving the uterine cervix of an 82-year-old woman as an incidental finding in a hysterectomy specimen. This unusual lesion should be differentiated from atypical glandular lesions of the cervix. Immunohistochemical studies confirm the histological suspicion of ectopic prostatic tissue.
\end{abstract}

\section{Keywords}

Uterine cervix · Prostatic tissue - Ectopia - Developmental anomaly $\cdot$ Immunohistochemistry ein muzinöser Borderline-Tumor vom endozervikalen Subtyp des linken Ovars diagnostiziert.

\section{Pathologisch-anatomische Befunde}

Nach der Aufarbeitung des Hysterektomiepräparates fand sich im Bereich der Transformationszone der Zervix eine auffällige Läsion ( $\bullet$ Abb. 1a, b). Diese wies eine kribriforme Architektur auf und bestand aus 2 Komponenten einer glandulären, deren Zellen ein auffällig vakuolisiertes Zytoplasma besaßen ( $\mathbf{0}$ Abb. 1b) und einer plattenepithelartigen Proliferation von Basalzellen ( $\bullet$ Abb. 1a).

In einem weiteren Herd fand sich eine Prädominanz der plattenepithelialen Komponente (• Abb. 2). Insbesondere die glandulären Zellen zeigten eine kräftige Immunexpression für prostataspezifisches Antigen (PSA) und prostataspezifische saure Phosphatase (PSAP; - Abb. 3a-c).

\section{Diskussion}

Ektopes Prostatagewebe bei der Frau wurde in der Literatur im Wesentlichen in Ovarialteratomen [2, 9] beschrieben, in einem Fall war dieses sogar mit einem "prostatischen“ Adenokarzinom des Ovars vergesellschaftet [2]. Andere Adenokarzinome vom „prostatischen Subtyp“ wurden in der Vagina beobachtet [1, 7]. Diese gehen mutmaßlich von den periurethral gelegenen Skene-Drüsen (Glandulae paraurethrales) aus, die als Rudimente des Wolff-Ganges (Ductus mesonephricus) entwicklungsgeschichtlich die weiblichen Homologe der männlichen Prostata sind und daher auch die entsprechenden histologischen und immunphänotypischen Charakteristika der Prostata aufweisen [8].

Da ektopes Prostatagewebe in der Cervix uteri dem Pathologen eher nicht vertraut ist, wird es vermutlich nur selten wahrgenommen $[5,6,10]$. Die wenigen bisher beschriebenen Fälle [3, 4, 5, 6, 10] waren sämtlich Zufallsbefunde nach Drahtschlingenexzision (LEEP, LLETZ), Konisation, oder Hysterektomie. Das Prostatagewebe liegt im Zervixstroma, ohne Beziehung zur Oberfläche. Charakteristischerweise finden sich kribriforme glanduläre Strukturen, die von einer zweireihigen Zellschicht gebildet werden. Fast immer finden sich in unterschiedlicher Ausprägung plattenepithelartige Areale, welche die glandulären Anteile weitgehend verdrängen können. Plattenepitelartige Metaplasien sind für die männliche Prostata ungewöhnlich, werden dort aber als Folge von Östrogenbehandlungen beobachtet. Eine maligne Entartung von Prostatagewebe in der Cervix uteri ist bisher nicht beschrieben worden.

Die Ätiologie von ektopem Prostatagewebe in der Zervix ist nicht eindeutig geklärt. Als wahrscheinlichste Hypothese wird eine embryonale Entwicklungsanomalie mit versprengtem Gewebe der Skene-Drüsen vertreten $[4,5,6]$. Ein weiterer embryologischer Ansatz interpretiert die ektopen Gewebsinseln als atypisch lokalisierte Reste des Wolff-Ganges. In einigen Fällen scheinen Androgene in der Ätiologie bzw. als begünstigende Kofaktoren eine Rolle zu spielen $[3,5,6]$, so dass die Entwicklung von Prostatagewebe auch im Sinne metaplastischer Veränderungen vorbestehender endozervikaler Drüsen gesehen wurde [3].

Ektopes Prostagewebe sollte von atypischen glandulären Läsionen in der Cervix uteri abgegrenzt werden. Das Fehlen von ausgeprägten nukleären Atypien, Mitosen sowie Apoptosen und letztendlich die immunhistochemischen Zusatzuntersuchungen mit PSA und PSAP erlauben die korrekte Diagnose. In den seltenen Fällen, in denen ektopes Prostagewebe PSA/PSAP-negativ ist, können CD 10 und Androgenrezeptor (AR) als zusätzliche immunhistochemische Marker eingesetzt werden [5].

\section{Fazit für die Praxis}

Ektopes Prostatagewebe kann in seltenen Fällen auch in der Cervix uteri lokalisiert sein und sollte daher bei der Differenzialdiagnose glandulärer Läsionen dieses Organs berücksichtigt werden. Die charakteristischen histomorphologischen Merkmale sowie immunhistochemische Zusatzuntersuchungen sichern die Diagnose. 


\section{Korrespondierender Autor}

PD Dr. G. Singer

Institut für Pathologie, Universitäts-Spital Schönbeinstraße 40, 4031 Basel, Schweiz gsinger@uhbs.ch

Interessenkonflikt. Es besteht kein Interessenkonflikt. Der korrespondierende Autor versichert, dass keine Verbindungen mit einer Firma, deren Produkt in dem Artikel genannt ist, oder einer Firma, die ein Konkurrenzprodukt vertreibt, bestehen. Die Präsentation des Themas ist unabhängig und die Darstellung der Inhalte produktneutral.

\section{Literatur}

1. Dodson MK, Cliby WA, Keeney GL et al. (1994) Skene's gland adenocarcinoma with increased serum level of prostate-specific antigen. Gynecol Oncol 55: 304-307

2. Halabi M, Oliva E, Mazal PR et al. (2002) Prostatic tissue in mature cystic teratomas of the ovary: a report of four cases, including one with features of prostatic adenocarcinoma, and cytogenetic studies. Int J Gynecol Pathol 21: 261-267

3. Kim KR, Park KH, Kim JW et al. (2004) Transitional cell metaplasia and ectopic prostatic tissue in the uterine cervix and vagina in a patient with adrenogenital syndrome: report of a case suggesting a possible role of androgen in the histogenesis. Int J Gynecol Pathol 23: 182-187

4. Larraza-Hernandez O, Molberg KH, Lindberg G, Albores-Saavedra J (1997) Ectopic prostatic tissue in the uterine cervix. Int J Gynecol Pathol 16: 291293

5. McCluggage WG, Ganesan R, Hirschowitz L et al. (2006) Ectopic prostatic tissue in the uterine cervix and vagina: report of a series with a detailed immunohistochemical analysis. Am J Surg Pathol 30: 209-215

6. Nucci MR, Ferry JA, Young RH (2000) Ectopic prostatic tissue in the uterine cervix: a report of four cases and review of ectopic prostatic tissue. Am J Surg Pathol 24: 1224-1230

7. Pongtippan A, Malpica A, Levenback C et al. (2004) Skene's gland adenocarcinoma resembling prostatic adenocarcinoma. Int J Gynecol Pathol 23: 7174

8. Tepper SL, Jagirdar J, Heath D, Geller SA (1984) Homology between the female paraurethral (Skene's) glands and the prostate. Immunohistochemical demonstration. Arch Pathol Lab Med 108: 423425

9. Vadmal M, Hajdu SI (1996) Prostatic tissue in benign cystic ovarian teratomas. Hum Pathol 27: 428-429

10. Wallace C, Creager AJ, Cappellari JO, Bergman S (2001) Ectopic prostatic tissue in the uterine cervix. Am J Surg Pathol 25: 1215-1216
Europäische Akademie legt Forschungsbericht $2005 / 2006$ vor

Die Europäische Akademie GmbH stellt in ihrem neuesten Forschungsbericht (Research Report) ihre Aktivitäten der Jahre 2005/2006 vor. Der Bericht dokumentiert die interdisziplinäre Arbeit der „Europäischen Akademie zur Erforschung von Folgen wissenschaftlich-technischer Entwicklungen.

Die Europäische Akademie zur Erforschung von Folgen wissenschaftlich-technischer Entwicklungen Bad Neuenahr-Ahrweiler gGmbH wurde 1996 vom Land Rheinland-Pfalz und dem Deutschen Zentrum für Luft- und Raumfahrt e.V. (DLR) gegründet. Wissenschaftlichinterdisziplinäre Arbeitsgruppen widmen sich der Erforschung und Beurteilung von Folgen wissenschaftlich-technischer Entwicklungen für das individuelle und soziale Leben des Menschen und seine natürliche Umwelt. In wissenschaftlicher Unabhängigkeit führt die Akademie einen Dialog mit Wirtschaft, Kultur, Politik und Gesellschaft. Damit will sie zu einem rationalen Umgang der Gesellschaft mit Folgen wissenschaftlich-technischer Entwicklungen beitragen.

In der Zeit 2005/2006 vollendete und präsentierte die Europäische Akademie fünf Projekte und startete sechs neue Forschungsgruppen. Zu den in den vergangenen zwei Jahren abgeschlossenen Studien gehören "Intervening in the Brain. Changing Psyche and Society ", ,'Leben mit Lärm? Risikobeurteilung und Regulation des Umgebungslärms im Verkehrsbereich", ",Organmangel. Ist der Tod auf der Warteliste unvermeidbar? ${ }_{, \text {, }} \mathrm{Na}$ notechnology. Assessment and Perspectives" sowie „Enabling Social Europe".

In Rahmen der Studie „Intervening in the Brain. Changing Psyche and Society" beispielsweise hat sich eine interdisziplinäre Projektgruppe, bestehend aus Neurowissenschaftlern, Medizinern, Philosophen und einem Juristen, aus erster Hand kritisch über derzeitige Möglichkeiten und Entwicklungsperspektiven in den Bereichen Psychopharmakologie, Neurotransplantation, Neuroprothetik und elektrische Stimulationstechniken auseinandergesetzt.

Die Zeit, nicht nur die medizinischen Möglichkeiten zu erforschen, sondern auch die gesellschaftlichen Veränderungen, die solche
Therapien nach sich ziehen können, ist längst angebrochen. Entsprechend schwankt die Debatte über neue Möglichkeiten der Einflussnahme auf das Gehirn zwischen euphorischen Vorwegnahmen neuer Therapieoptionen und finsteren Befürchtungen bezüglich selbstentfremdeter Individuen.

Die neue Studie der Europäischen Akademie $\mathrm{GmbH}$ bietet in dieser Situation eine rationale Orientierungshilfe. Die Experten ziehen praxisbezogene Schlussfolgerungen, die einen verantwortbaren Umgang mit den neuen Eingriffen in das Gehirn vorbereiten soll.

Originalpublikation: Merkel R, Boer G, Fegert J et al. (2007) Intervening in the Brain: Changing Psyche and Society. Springer, Berlin Heidelberg New York

Quelle: Europäische Akademie GmbH, www.ea-aw.de 